\title{
ON THE EXISTENCE AND STABILITY OF SOLUTIONS TO STOCHASTIC EQUILIBRIUM PROBLEMS
}

\author{
Lam Quoc Anh ${ }^{1}$, Nguren Xuan $\mathrm{Hai}^{2}$, Kien Trung Nguyen ${ }^{1, *}$, \\ Nguyen Hong QuAn ${ }^{2}$ And DAng Thi My VAN ${ }^{3}$
}

\begin{abstract}
In this paper we consider stochastic equilibrium problems involving parameter of probability measures. Employing the fixed point theorem of Knaster, Kuratowski, Mazurkiewicz, and Fan (KKM-Fan), conditions for the existence of solutions to such problems are established. We then propose new metric concepts on the underlying stochastic spaces and study some properties corresponding to these metrics. Afterwards, we study sufficient conditions for the solution mappings of such problems, that are closed, upper (lower) semicontinuous and continuous with respect to the mentioned metrics. Finally, the special cases of stochastic optimization problems are taken into account as the applications.
\end{abstract}

Mathematics Subject Classification. 49K40, 49K45, 90C15, 90C31.

Received July 8, 2019. Accepted December 22, 2019.

\section{INTRODUCTION}

In many real life situations, we usually have to make decisions in a reasonably optimal way under uncertain conditions, which may exist in the case we cannot list all possible outcomes and/or cannot assign exact probabilities to the various outcomes. For example, the future demands or future interest rates for some products are not available at the time we make decisions. Another example concerning the planning of water resources to release into the channels or canals of a river system in each period. Then one of the most important parameters of this model is the rainfall amounts at various times, which is predicted based on the estimation of historical records and is therefore uncertain. Various settings similar to these two examples can be also modelled as the stochastic optimization problems. Therefore, the investigation of stochastic optimization has become one of the interesting and important topic in optimization theory and applications, including existence conditions $[27,31,32,34,40,43]$, stability conditions $[12,15,37]$ and solution methods [3, 14, 45]. This fact has inspired many mathematicians to study various generalized stochastic problems related to optimization with numerious applications to real-world problems, such as stochastic variational inequalities [4,7,26,33,42], stochastic Nash

Keywords. Stochastic equilibrium problems, stochastic optimization, probabilistic metric spaces, existence and stability conditions, closedness and upper (lower) semicontinuity/continuity.

1 Department of Mathematics, Teacher College, Can Tho University, Can Tho, Vietnam.

2 Department of Scientific Fundamentals, Posts and Telecommunications Institute of Technology of Vietnam, Ho Chi Minh City, Vietnam.

3 Department of Mathematics, Cantho College, Can Tho, Vietnam.

* Corresponding author: trungkien@ctu.edu.vn 
equilibrium problems $[1,25,41,44]$, stochastic mathematical programs with equilibrium constraints $[8,22,35]$ with numerious applications to real-world problems.

Study of equilibrium phenomena encountered in engineering, physics, chemistry or economics plays an important role in the theory of nonlinear analysis and optimization. There are many models which were proposed for containing various kinds of these equilibria such as inequalities of Ky Fan [9], Takahashi [36], and Muu [19]. The name "Equilibrium Problem" was first considered by Muu and Oettli [20], since it coincides with finding the equilibrium point of an optimization problem under certain conditions. Then this model was shown to contain various important problems related to optimization, including complementarity problems, Nash equilibrium, traffic network problems, etc. [5]. Therefore, properties of solutions to equilibrium problem and its related-problems are worthwhile topics to discuss, and they have been studied by many mathematicians all over the world (see e.g., $[2,11,38,39]$ and the references therein). To the best of our knowledge, conditions for the existence and stability of solutions to stochastic equilibrium problems have not been under investigation so far despite of their importance in practice.

In this paper we aim to introduce stochastic equilibrium problems as well as to study the existence and stability conditions for such problems. Firstly, using KKM-Fan fixed point theorem, we study conditions for the existence of solutions to such problems. We then propose various metrics on stochastic spaces and employ them to establish sufficient conditions for solution mappings to such problems to be both closed, upper semicontinuous or continuous. For applications, we discuss the obtained results in order to deal with some special cases of the stochastic optimization problems.

The rest of this paper is organized as follows. Section 2 presents the setting of parametric stochastic equilibrium problems and some preliminary results used in the sequel. Section 3 is devoted to the existences conditions for stochastic equilibrium problems. In Section 4, we introduce some metrics on the referenced stochastic spaces and discuss some results involving the equipped metrics, which are needed in the sequel. In Section 5 , we study sufficient conditions for the solution mappings to such problems to be closed and upper semicontinuous with respect to the metrics proposed in the previous section. For applications, we consider in the last section special cases of stochastic optimizations.

\section{PRELIMinaRies}

Let $X$ be a nonempty, closed and bounded subset of $\mathbb{R}^{k} ; \Omega$ be a nonempty, closed subset of $\mathbb{R}^{l} ; \mathcal{P}(\Omega)$ be the set of all Borel probability measures on $\Omega$; and $M$ be a nonempty subset of $\mathcal{P}(\Omega)$. Furthermore, let $f: \Omega \times X \times X \rightarrow \overline{\mathbb{R}}=\mathbb{R} \cup\{ \pm \infty\}$ be a single valued mapping such that $f(\cdot, x, y)$ is a measurable function for $x, y \in X$ and $K: M \rightrightarrows X$ be a multivalued mapping. We define a stochastic equilibrium problem involving the parameter $\mu \in M$ as follows.

$\left(\mathrm{SEP}_{\mu}\right) \quad$ Find $\bar{x} \in K(\mu)$ such that

$$
\mathbb{E}_{\mu}[f(\omega, \bar{x}, y)] \leq 0, \forall y \in K(\mu) .
$$

Here, $\mathbb{E}_{\mu}[f(\omega, x, y)]=\int_{\Omega} f(\omega, x, y) \mu(\mathrm{d} \omega)$ is the expectation of $f(\cdot, x, y)$ with respect to the measure $\mu$. Denote by $\operatorname{Sol}\left(\mathrm{EP}_{\mu}\right)$ the solution set to $\left(\mathrm{SEP}_{\mu}\right)$, we get

$$
\operatorname{Sol}\left(\operatorname{SEP}_{\mu}\right)=\left\{x \in X: x \in K(\mu), \int_{\Omega} f(\omega, x, y) \mu(\mathrm{d} \omega) \leq 0, \quad \forall y \in K(\mu)\right\}
$$

A multivalued mapping $S: M \subset \mathcal{P}(\Omega) \rightrightarrows X$ identified as $S(\mu)=\operatorname{Sol}\left(\mathrm{SEP}_{\mu}\right)$ is called the solution mapping to the reference problem. As mentioned in the previous section, this paper aims to study the nonempty values, closedness and upper continuity of $S$ regarding the probabilistic parameter $\mu$.

For better illustration, we consider some special cases of $\left(\mathrm{SEP}_{\mu}\right)$ as follows.

(a) If $f(\omega, x, y)=\varphi(\omega, y)-\varphi(\omega, x)$ where $\varphi: \Omega \times X \rightarrow \overline{\mathbb{R}}$, then $\left(\mathrm{SEP}_{\mu}\right)$ is the classical optimization problem. 
(b) If $f(\omega, x, y)=\langle g(\omega, x), x-y\rangle$ where $g: \Omega \times X \rightarrow \mathbb{R}^{n}$ is a map, then ( $\mathrm{SEP}_{\mu}$ ) can reduce to a stochastic variational inequality.

In the context of Nash games with convex player problems, the problem $\left(\mathrm{SEP}_{\mu}\right)$ could be transferred to a stochastic variational inequality where $f(\omega, x, y)=\langle F(\omega, x), x-y\rangle$ and $F(\omega, x)$ denotes the concatenated gradient map.

We now recall some concepts and properties that will be used thereafter.

Definition 2.1. Let $F: M \rightrightarrows X$ be a multivalued mapping.

(a) The inverse image of a subset $A$ of $M$ by $F$ is defined by

$$
F^{-1}(A)=\{x \in M: F(x) \cap A \neq \emptyset\} .
$$

(b) $F$ is said to be closed if its graph given as

$$
\text { Graph } F=\{(\mu, x) \in M \times X: x \in F(\mu)\}
$$

is a closed subset of $M \times X$.

(c) $F$ is said to be lower semicontinuous (lsc) at $\mu_{0}$ if for any open set $U \subset X$ with $F\left(\mu_{0}\right) \cap U \neq \emptyset$, there exists an open neighborhood $N$ of $\mu_{0}$ such that $F(\mu) \cap U \neq \emptyset$ for all $\mu \in N$.

Equivalently, $F$ is lsc at $\mu_{0}$ if for all sequences $\left\{\mu_{n}\right\}_{n=1}^{\infty}$ converging to $\mu_{0}$ and for all $x \in F\left(\mu_{0}\right)$, there exist $x_{n} \in F\left(\mu_{n}\right)$ such that $\left\{x_{n}\right\}_{n=1}^{\infty}$ converges to $x$.

(d) $F$ is said to be upper semicontinuous (usc) at $\mu_{0}$ if for any open set $U \supset F\left(\mu_{0}\right)$, there exists an open neighborhood $N$ of $\mu_{0}$ such that $U \supset F(N)$.

Finally, $F$ is called continuous at $\mu_{0}$ if it is both usc and lsc at $\mu_{0}$. We say that $F$ is lsc (usc, continuous) if it is lsc (usc, continuous) at every points of $M$.

We denote by $\|\cdot\|$ the Euclidean norm on $\mathbb{R}^{k}$. For $x \in X$ and $A \subset X$, denote by $d(x, A)=\inf _{y \in A}\|x-y\|$ and $\mathbb{B}(A, r)=\{x \in X: d(x, A)<r\}$. If $F$ has compact values, then $F$ is usc at $\mu_{0}$ if and only if for any $r>0$, there exists $\delta>0$ such that $F\left(\mathbb{B}\left(\mu_{0}, \delta\right)\right) \subset \mathbb{B}\left(F\left(\mu_{0}\right), r\right)$, where $\mathbb{B}\left(F\left(\mu_{0}\right), r\right):=\left\{x \in X: d\left(x, F\left(\mu_{0}\right)\right)<r\right\}$.

Definition 2.2. Let $F$ be as in Definition 2.1.

(a) The mapping $F$ is called Hausdorff lower semicontinuous (H-lsc) at $\mu_{0}$ if for any neighborhood $B$ of the origin in $\mathbb{R}^{n}$, there exists an open neighborhood $N$ of $\mu_{0}$ such that $F\left(\mu_{0}\right) \subset F(\mu)+B$ for all $\mu \in N$.

(b) The mapping $F$ is called Hausdorff upper semicontinuous (H-usc) at $\mu_{0}$ if for any neighborhood $B$ of the origin in in $\mathbb{R}^{n}$, there exists an open neighborhood $N$ of $\mu_{0}$ such that $F(\mu) \subset F\left(\mu_{0}\right)+B$ for all $\mu \in N$.

$F$ is called Hausdorff continuous at $\mu_{0}$ if it is both Hausdorff upper semicontinuous and Hausdorff lower semicontinuous at $\mu_{0}$.

Obviously, the Hausdorff upper semicontinuity/or Hausdorff lower semicontinuity is weaker/or stronger than the upper/or lower semi-continuity, respectively, and they are equivalent if the underlying mapping has compact values.

The Hausdorff continuity is often described in the Hausdorff metric meaning as follows.

For two closed subsets $A$ and $B$ of $X$, the Hausdorff distance between these subsets is

$$
\mathcal{H}(A, B)=\max \left\{\sup _{x \in A} d(x, B), \sup _{y \in B} d(y, A)\right\},
$$

which is indeed a metric as $X$ is bounded. Then, $F$ is Hausdorff continuous at $\mu_{0}$ if and only if $\mathcal{H}\left(F(\mu), F\left(\mu_{0}\right)\right) \rightarrow$ 0 as $\mu \rightarrow \mu_{0}$.

A single valued mapping $h: X \rightarrow \mathbb{R}$ is called upper semicontinuous (lower semicontinuous) if for all $\alpha \in \mathbb{R}$, the upper level set $\{x \in X: h(x) \geq \alpha\}$ (the lower level set $\{x \in X: h(x) \leq \alpha\}$, respectively) is closed on $X$. 
Definition 2.3 (See [28]). Let $g: \Omega \times X \rightarrow \mathbb{R}$. The mapping $g$ is said to be random lower semicontinuous if the corresponding epigraph multivalued mapping $\omega \rightarrow \operatorname{epi} g(\omega, \cdot)$ has closed measurable values for each $\omega \in \Omega$.

Lemma 2.4 (See [28]). Let $g$ be as in Definition 2.3. If $g$ is random lower semicontinuous then the folowing statements hold

(i) for each $\omega \in \Omega$ and $x \in X, g(\cdot, x)$ is measurable and $g(\omega, \cdot)$ is lower semicontinuous.

(ii) the mapping $\omega \rightarrow \inf _{x \in X} g(\omega, x)$ is well defined and measurable.

\section{EXISTENCE CONDITIONS}

In this section we assume that $K$ has nonempty, convex and compact values on $M$, and $f(\cdot, x, y)$ is measurable for all $(x, y) \in X \times X$ and $f(\omega, \cdot, \cdot)$ is an equilibrium function for a.e. $\omega \in \Omega$ on K, i.e., for a.e. $\omega \in \Omega$, $f(\omega, x, x)=0$ for all $x \in X$. In what follows, we investigate sufficient conditions for the existence of solutions to $\left(\mathrm{SEP}_{\mu}\right)$.

Firstly, we propose and recall some basic concepts and their properties used in the sequel.

Definition 3.1. Let $A \subset \mathbb{R}^{k}$ be a nonempty and convex subset, and $\varphi: A \rightarrow \overline{\mathbb{R}}$ be a single valued mapping. Then, $\varphi$ is said to be 0 -lower level quasiconcave on $A$ if for each $x_{1}, x_{2} \in A$ and $\lambda \in[0,1]$ and $\varphi\left[\lambda x_{1}+(1-\lambda) x_{2}\right] \leq 0$ then $\varphi\left(x_{1}\right) \leq 0$ or $\varphi\left(x_{2}\right) \leq 0$.

Remark 3.2. If $\varphi$ is quasiconcave on $A$, i.e., $\min \left\{\varphi\left(x_{1}\right), \varphi\left(x_{2}\right)\right\} \leq \varphi\left[\lambda x_{1}+(1-\lambda) x_{2}\right]$ for all $x_{1}, x_{2} \in A$ and $\lambda \in[0,1]$, then it is 0 -lower level quasiconcave on $A$.

Now we introduce equivalent statements of the 0-lower level quasiconcavity. Because the proofs are elementary, we would like to omit them.

Lemma 3.3. Let $\varphi, A$ be as in Definition 3.1. The following conditions are equivalent to each other.

(a) $\varphi$ is 0 -lower level quasiconcave on $A$.

(b) For all $\left\{x_{1}, x_{2}, \ldots, x_{n}\right\} \subset A$ and $x \in \operatorname{conv}\left\{x_{1}, x_{2}, \ldots, x_{n}\right\}$ satisfying $\varphi(x) \leq 0$, then there exists $i \in$ $\{1,2, \ldots, n\}$ such that $\varphi\left(x_{i}\right) \leq 0$, where $\operatorname{conv}\left\{x_{1}, x_{2}, \ldots, x_{n}\right\}$ is the convex hull of $\left\{x_{1}, x_{2}, \ldots, x_{n}\right\}$, i.e., $\left\{x_{1}, x_{2}, \ldots, x_{n}\right\}=\left\{x \in \mathbb{R}^{n}: x=\sum_{i=1}^{n} \lambda_{i} x_{i}, \lambda_{i} \geq 0, \sum_{i=1}^{n} \lambda_{i}=1\right\}$.

(c) The set $\{x \in A: \varphi(x)>0\}$ is convex.

Based on Lemma 3.3, the following example shows that the converse of Remark 3.2 is not true.

Example 3.4. Let $A=\mathbb{R}^{k}$ and $\varphi: A \rightarrow \overline{\mathbb{R}}$ be defined by $\varphi(x)=1+\|x\|$ for all $x \in A$. Then, the set $\{x \in A: \varphi(x)>0\}$ is equal to $\mathbb{R}^{k}$, and hence it is convex. Applying Lemma 3.3, we conclude that $\varphi$ is 0 lower level quasiconcave on $A$. However, for $x_{1}=(-2,0, \ldots, 0), x_{2}=(2,0, \ldots, 0) \in A$ and $\lambda=\frac{1}{2}$, we have $3=\min \left\{\varphi\left(x_{1}\right), \varphi\left(x_{2}\right)\right\}>\varphi\left[\lambda x_{1}+(1-\lambda) x_{2}\right]=1$, so $\varphi$ is not quasiconcave on $A$.

Now we recall the concept of KKM mapping introduced by Knaster, Kuratowski and Mazurkiewicz in 1929.

Definition 3.5 (See [18]). Let $X$ be a subset of topological vector space $Y$. A map $G: X \rightrightarrows Y$ is called a KKM mapping on $X$ if for each subset $\left\{x_{1}, \ldots, x_{n}\right\} \subset X$, then $\operatorname{conv}\left\{x_{1}, x_{2}, \ldots, x_{n}\right\} \subset \cup_{i=1}^{n} G\left(x_{i}\right)$.

The following result is usually known as the KKM-Fan fixed point theorem.

Lemma 3.6 (See [10], Cor. 1). Let $X$ be an arbitrary set in a topological vector space $Y$, and let $G: X \rightrightarrows Y$ be a KKM mapping with closed values on $X$. If $G(x)$ is compact for at least one $x \in X$, then $\cap_{x \in X} G(x) \neq \emptyset$.

Let $\mu \in M$ be fixed, we study sufficient conditions for the existence of solutions to $\left(\mathrm{SEP}_{\mu}\right)$.

Theorem 3.7. Assume that

(i) For each $\omega \in \Omega$ and $y \in K(\mu)$, the set $\left\{x \in K(\mu): \mathbb{E}_{\mu}[f(\omega, x, y)] \leq 0\right\}$ is closed; 
(ii) for each $x \in K(\mu), f(\omega, x, \cdot)$ is 0-lower level quasiconcave for a.e. $\omega \in \Omega$ on $K(\mu)$.

Then, the problem $\left(\mathrm{SEP}_{\mu}\right)$ is feasible.

Proof. For $x, y \in K(\mu)$, setting

$$
\begin{aligned}
& P(x)=\left\{z \in K(\mu): E_{\mu}[f(\omega, x, y)]>0\right\}, \\
& \Phi(x)=K(\mu) \cap P(x), \\
& Q(y)=K(\mu) \backslash \Phi^{-1}(y),
\end{aligned}
$$

we have

$$
\begin{aligned}
Q(y) & =\left\{x \in K(\mu): x \notin \Phi^{-1}(y)\right\}=\{x \in K(\mu): y \notin \Phi(x)\} \\
& =\{x \in K(\mu): y \notin P(x)\}=\left\{x \in K(\mu): x \notin P^{-1}(y)\right\} \\
& =K(\mu) \backslash P^{-1}(y)
\end{aligned}
$$

where the subsets $\Phi^{-1}(y)$ and $P^{-1}(y)$ are the inverse images of $y$ by the multivalued mappings $\Phi$ and $P$, respectively.

We first prove that $Q$ is a KKM mapping on $K(\mu)$. Suppose, we proceed by contradiction and assume that there exist a subset $\left\{y_{1}, y_{2}, \ldots, y_{n}\right\} \subset K(\mu)$ and $\hat{x} \in \operatorname{conv}\left\{y_{1}, y_{2}, \ldots, y_{n}\right\}$ such that $\hat{x} \notin \cup_{i=1}^{n} Q\left(y_{i}\right)$. Then, for all $i \in\{1,2, \ldots, n\}, \hat{x} \notin Q\left(y_{i}\right)$, i.e., $y_{i} \in \Phi(\hat{x})$. Hence, $y_{i} \in P(\hat{x})$ for all $i \in\{1,2, \ldots, n\}$. Consequently, $\mathbb{E}_{\mu}\left[f\left(\omega, \hat{x}, y_{i}\right)\right]>0$ for all $i \in\{1,2, \ldots, n\}$. This contradicts assumption (ii) and the equilibrium assumption of $f$. So, $Q$ is a KKM mapping on $K(\mu)$.

It follows from assumption (i) that $Q(y)$ is a closed subset as

$$
Q(y)=K(\mu) \backslash P^{-1}(y)=\left\{x \in K(\mu): \mathbb{E}_{\mu}[f(\omega, x, y)] \leq 0\right\} .
$$

By the boundedness of $X, Q(y)$ is compact. Applying Lemma 3.6, there exists $\bar{x} \in X$ such that

$$
\bar{x} \in \cap_{y \in K(\mu)} Q(y)=K(\mu) \backslash\left[\cup_{y \in K} \Phi^{-1}(y)\right] .
$$

This also means that $\bar{x} \in K(\mu)$ and $\bar{x} \notin \Phi^{-1}(y)$ for all $y \in K(\mu)$, i.e., $y \notin P(\bar{x})$ for all $y \in K(\mu)$. Hence, we conclude that $\mathbb{E}_{\mu}[f(\omega, \bar{x}, y)] \leq 0$ for all $y \in K(\mu)$. Therefore, $\bar{x}$ is a solution to $\left(\mathrm{SEP}_{\mu}\right)$.

Remark 3.8. For a mapping $g: \Omega \times X \rightarrow \mathbb{R}^{k}$, we define $F(x)=\mathbb{E}_{\mu}[g(\omega, x)]$ for $x \in X$ and $\omega \in \Omega$, where $\mu \in M$ is given. A stochastic variational inequality (SVI) mentioned in [26] is as follows.

$\left(\mathrm{SVI}_{\mu}\right)$ Find $\bar{x} \in X$ such that

$$
\langle F(\bar{x}), \bar{x}-y\rangle \leq 0, \forall y \in X .
$$

Setting $f(\omega, x, y)=\langle g(\omega, x), x-y\rangle$, then the problem $\left(\mathrm{SEP}_{\mu}\right)$ reduces to above stochastic variational inequality. For $\left(\mathrm{SVI}_{\mu}\right)$, assumption (ii) of Theorem 3.7 is clearly satisfied, employing this theorem, we get the following result.

Corollary 3.9. $\left(\mathrm{SVI}_{\mu}\right)$ is feasible if the set $M=\left\{x \in X: \mathbb{E}_{\mu}[f(\omega, x, y)] \leq 0\right\}$ is closed for each $y \in X$ and $\omega \in \Omega$.

Because our approach is different from that of [26], Corollary 3.9 cannot be compared to Proposition 3 in [26].

Finally, we employ a regularity property of $f$ to replace assumption (i) in Theorem 3.7 as follows.

Theorem 3.10. Suppose that assumption (ii) of Theorem 3.7 is satisfied and assume further that, for each $y \in K(\mu)$, the mapping $f(\cdot, \cdot, y)$ is random lower semicontinuous on $\Omega \times K(\mu)$. Then, the problem $\left(\mathrm{SEP}_{\mu}\right)$ is feasible. 
Proof. We need to prove that the assumption (i) of Theorem 3.7 holds true. Taking

$$
M=\left\{x \in K(\mu): \mathbb{E}_{\mu}[f(\omega, x, y)] \leq 0\right\},
$$

and an arbitrary sequence $\left\{x_{n}\right\} \subset M$ converging to $x^{*}$, we will show that $x^{*}$ belongs to $M$. Because $f$ is random lower semicontinuous, Lemma 2.4(i) implies that for each $\omega \in \Omega$ and $y \in K(\mu), f\left(\cdot, x^{*}, y\right)$ is measurable, $f(\omega, \cdot, y)$ is lower semicontinuous, and hence it follows from [31] (Appendix) that $E_{\mu}[f(\omega, x, y)]$ is well-defined. Also,

$$
\mathbb{E}_{\mu}\left[f\left(\omega, x^{*}, y\right)\right] \leq \mathbb{E}_{\mu}\left[\liminf _{n \rightarrow \infty} f\left(\omega, x_{n}, y\right)\right] .
$$

Putting $g(\omega, y)=\min _{x \in K(\mu)} f(\omega, x, y)$. Because $f$ is random lower semicontinuous, Lemma 2.4(ii) implies that the function $g$ is well defined and measurable, and hence the Fatou's lemma (see e.g., [32], Thm. 58) can use to get

$$
\mathbb{E}_{\mu}\left[f\left(\omega, x^{*}, y\right)\right] \leq \mathbb{E}_{\mu}\left[\liminf _{n \rightarrow \infty} f\left(\omega, x_{n}, y\right)\right] \leq \liminf _{n \rightarrow \infty} \mathbb{E}_{\mu}\left[f\left(\omega, x_{n}, y\right)\right] \leq 0,
$$

or equivalently, $x^{*} \in M$. Thus, assumption (i) of Theorem 3.7 holds. Applying Theorem 3.7, we conclude that the solution set of $\left(\mathrm{SEP}_{\mu}\right)$ is nonempty.

\section{VARIOUS PROBABILISTIC METRIC SPACES}

To study the stability of $\left(\mathrm{SEP}_{\mu}\right)$, we introduce some metrics in the set of parameters with probability measure $\mathcal{P}(\Omega)$ and its subset $\mathcal{P}_{p}(\Omega)$. Noting that it follows from different goal of investigating the stochastic problems, various metrics on $\mathcal{P}(\Omega)$ and its subsets are introduced (see $[6,12,16,17,21,23,24,28-30]$ ). Among them is the Fortet-Mourier metric (see e.g., [23]): For $p \geq 1$, let

$$
\mathcal{P}_{p}(\Omega)=\left\{\mu \in \mathcal{P}(\Omega): \int_{\Omega}\|\omega\|^{p} \mu(\mathrm{d} \omega)<\infty\right\},
$$

the Fortet-Mourier metric is defined

$$
\zeta_{p}(\mu, \nu)=\sup _{g \in \mathcal{T}_{p}(\Omega)}\left|\int_{\Omega} g(\omega)(\mu-\nu)(\mathrm{d} \omega)\right|, \forall \mu, \nu \in \mathcal{P}_{p}(\Omega)
$$

where

$$
\mathcal{T}_{p}(\Omega)=\left\{g: \Omega \rightarrow \mathbb{R}:|g(\omega)-g(\bar{\omega})| \leq\|\omega-\bar{\omega}\| \max \left\{1,\|\omega\|^{p-1},\|\bar{\omega}\|^{p-1}\right\}, \forall \omega, \bar{\omega} \in \Omega\right\} .
$$

For more comprehensive details of this metric, we refer the reader to [13] and the references therein.

Now, we define the following sets used in the sequel.

$$
\begin{gathered}
\mathcal{K}=\{K: \mathcal{P}(\Omega) \rightrightarrows X: K \text { has closed values }\} \\
\mathcal{L}=\{f: \Omega \times X \times X \rightarrow \overline{\mathbb{R}}: \forall y \in X, f(\cdot, \cdot, y) \text { is random lower semicontinuous }\}
\end{gathered}
$$

For $\mu \in \mathcal{P}(\Omega)$ and $f \in \mathcal{L}$, let

$$
\Gamma_{f}(\mu, y)=\left\{x \in X: \int_{\Omega} f(\omega, x, y) \mu(\mathrm{d} \omega) \leq 0\right\} .
$$

Lemma 4.1. For $y \in X$, if $f(\cdot, \cdot, y)$ is random lower semicontinuous then $\Gamma_{f}(\mu, y)$ is compact on $X$ for all $\mu$.

Proof. Taking an arbitrary sequence $\left\{x_{n}\right\}_{n=1}^{\infty} \subset \Gamma_{f}(\mu, y)$ converging to $x \in X$, we prove that $x \in \Gamma_{f}(\mu, y)$. Because of the Fatou's lemma, we have

$$
\int_{\Omega} f(\omega, x, y) \mu(\mathrm{d} \omega) \leq \liminf _{n \rightarrow \infty} \int_{\Omega} f\left(\omega, x_{n}, y\right) \mu(\mathrm{d} \omega) \leq 0,
$$

i.e., $x \in \Gamma_{f}(\mu, y)$. Therefore, $\Gamma_{f}(\mu, y)$ is a closed set. Since $X$ is bounded, $\Gamma_{f}(\mu, y)$ is compact. 
Motivated by the Hausdorff metric on compact subsets, we define the functions $\rho$ and $\sigma_{p}$ on $\mathcal{P}(\Omega)$ and $\mathcal{P}_{p}(\Omega)$, respectively, as follows.

- For every $\mu, \nu \in \mathcal{P}(\Omega)$,

$$
\rho(\mu, \nu)=\sup _{K \in \mathcal{K}} \mathcal{H}(K(\mu), K(\nu))+\sup _{f \in \mathcal{L}} \sup _{y \in X} \mathcal{H}\left(\Gamma_{f}(\mu, y), \Gamma_{f}(\nu, y)\right) .
$$

- For every $\mu, \nu \in \mathcal{P}_{p}(\Omega)$,

$$
\sigma_{p}(\mu, \nu)=\sup _{K \in \mathcal{K}} \mathcal{H}(K(\mu), K(\nu))+\sup _{g \in \mathcal{T}_{p}(\Omega)}\left|\int_{\Omega} g(\omega)(\mu-\nu)(\mathrm{d} \omega)\right| .
$$

Because $X$ is bounded, we conclude that $K(\mu)$ and $\Gamma_{f}(\mu, y)$ are compact subsets of $X$. This together with the finite value of Fortet-Mourier metric, the functions $\rho$ and $\sigma_{p}$ are finite values. Employing properties of supremum of a set, the Hausdorff metric for compact sets and the Fortet-Mourier metric, $\rho$ and $\sigma_{p}$ are metrics on $\mathcal{P}(\Omega)$ and $\mathcal{P}_{p}(\Omega)$, respectively. Therefore, the parameter spaces of probability measure $(\mathcal{P}(\Omega), \rho),\left(\mathcal{P}_{p}(\Omega), \sigma_{p}\right)$ and $\left(\mathcal{P}_{p}(\Omega), \zeta_{p}\right)$ are metric spaces.

Thanks to the nice definitions of $\rho$ and $\sigma_{p}$, we get the following wonderful results.

Lemma 4.2. Let $R:(\mathcal{P}(\Omega), \rho) \rightrightarrows X$ and $T:\left(\mathcal{P}_{p}(\Omega), \sigma_{p}\right) \rightrightarrows X$ be multivalued mappings. If $R$ and $T$ have nonempty and closed values, then they are closed and lower semicontinuous.

Proof. Firstly, taking an arbitrary sequence $\left\{\left(\mu_{n}, y_{n}\right)\right\} \subset$ Graph $R$ converging to $(\mu, y)$, we show that $(\mu, y) \in$ Graph $R$. Because $\mathcal{H}\left(R\left(\mu_{n}\right), R(\mu)\right) \leq \rho\left(\mu_{n}, \mu\right)$ and $\rho\left(\mu_{n}, \mu\right) \rightarrow 0$ as $n \rightarrow+\infty$, we conclude that

$$
\mathcal{H}\left(R\left(\mu_{n}\right), R(\mu)\right) \rightarrow 0 \quad \text { as } n \rightarrow+\infty .
$$

Since $d(y, R(\mu))=\inf _{z \in R(\mu)}\|y-z\| \leq\left\|y_{n}-y\right\|+\mathcal{H}\left(R\left(\mu_{n}\right), R(\mu)\right) \rightarrow 0$, we get $d(y, R(\mu))=0$. As $R(\mu)$ is a closed subset of $X$, we have $y \in R(\mu)$. Therefore, $R$ is closed.

Next, we prove that $R$ is lsc. Let $\left\{\mu_{n}\right\} \subset \mathcal{P}(\Omega)$ be an arbitrary sequence converging to $\mu \in \mathcal{P}(\Omega)$, and $y \in R(\mu)$. For each $n$, as $R\left(\mu_{n}\right)$ is a nonempty, closed set and $X$ is bounded, there exists $y_{n} \in R\left(\mu_{n}\right)$ such that $\left\|y_{n}-y\right\|=d\left(y, R\left(\mu_{n}\right)\right)$. We have

$$
\begin{aligned}
\left\|y_{n}-y\right\| & =d\left(y, R\left(\mu_{n}\right)\right) \\
& \leq \sup _{y^{\prime} \in R(\mu)} d\left(y^{\prime}, R\left(\mu_{n}\right)\right) \\
& \leq \mathcal{H}\left(R\left(\mu_{n}\right), R(\mu)\right) \leq \rho\left(\mu_{n}, \mu\right) \rightarrow 0,
\end{aligned}
$$

and hence $y_{n} \rightarrow y$. Therefore, $R$ is lower semicontinuous.

Using the same arguments, the conclusions for $T$ in Lemma 4.2 are also obtained.

Unfortunately, the following example shows that for the metric space $\left(\mathcal{P}_{p}(\Omega), \zeta_{p}\right)$, the similar results as that of Lemma 4.2 do not hold.

Example 4.3. Let $\Omega=\mathbb{R}, X=[0,1], \bar{\mu} \in \mathcal{P}_{p}(\Omega)$ be fixed, $E:\left(\mathcal{P}_{p}(\Omega), \zeta\right) \rightrightarrows X$ defined by

$$
E(\mu)= \begin{cases}\{1\}, & \text { if } \mu=\bar{\mu}, \\ \{0\}, & \text { otherwise. }\end{cases}
$$

Then, $E$ is closed-valued, but it is not lsc. For this case, $E$ is a single-valued mapping, we will show that $E$ is discontinuous. Taking a sequence $\left\{\mu_{n}\right\}, \mu_{n}=\frac{n-1}{n} \bar{\mu}+\frac{1}{n} \hat{\mu}$ where $\hat{\mu} \in \mathcal{P}_{p}(\Omega)$ is given, then

$$
\zeta_{p}\left(\mu_{n}, \bar{\mu}\right)=\frac{1}{n} \sup _{g \in \mathcal{T}_{p}(\Omega)}\left|\int_{\Omega} g(\omega)(\hat{\mu}-\bar{\mu})(\mathrm{d} \omega)\right| .
$$

Because of the definitions of $\mathcal{T}_{p}(\Omega)$ and $\mathcal{P}_{p}(\Omega)$, the $\sup _{g \in \mathcal{T}_{p}(\Omega)}\left|\int_{\Omega} g(\omega)(\hat{\mu}-\bar{\mu})(\mathrm{d} \omega)\right|$ is finite, and hence $\zeta_{p}\left(\mu_{n}, \bar{\mu}\right) \rightarrow$ 0 as $n \rightarrow+\infty$. Because $E\left(\mu_{n}\right)=0 \nrightarrow E(\bar{\mu})=1, E$ is not continuous at $\bar{\mu}$. 
Consider a map $H: \mathcal{P}_{p}(\Omega) \rightrightarrows X$ defined by

$$
H(\mu)=\left\{x \in X: \int_{\Omega} m_{i}(\omega, x) \mu(\mathrm{d} \omega) \leq 0, i=1,2, \ldots, l\right\}
$$

where $m_{i}: \Omega \times X \rightarrow \mathbb{R}$ satisfy: for $x \in X, m_{i}(\cdot, x) \in \mathcal{T}_{p}(\Omega)$, and $m_{i}$ are random lower semicontinuous, $i=1,2, \ldots, l$. Fatou's lemma shows obviously that $H$ has closed values.

Lemma 4.4. The map $H:\left(\mathcal{P}_{p}(\Omega), \zeta_{p}\right) \rightrightarrows X$ defined by (4.1) is closed and upper semicontinuous.

Proof. For any sequence $\left\{\mu_{n}\right\}$ converging to $\mu$ in $\left(\mathcal{P}_{p}(\Omega), \zeta_{p}\right)$, let $\left\{y_{n}\right\}$ be a sequence in $X$ such that for all $n$, $y_{n} \in H\left(\mu_{n}\right)$ and $y_{n} \rightarrow y$ for $n \rightarrow \infty$. By Fatou's lemma, for $i=1,2, \ldots, l$, we have

$$
\begin{aligned}
\int_{\Omega} m_{i}(\omega, y) \mu(\mathrm{d} \omega) & \leq \liminf _{n \rightarrow \infty} \int_{\Omega} m_{i}\left(\omega, y_{n}\right) \mu(\mathrm{d} \omega) \\
& \leq \liminf _{n \rightarrow \infty}\left[\left|\int_{\Omega} m_{i}\left(\omega, y_{n}\right)\left(\mu_{n}-\mu\right)(\mathrm{d} \omega)\right|+\int_{\Omega} m_{i}\left(\omega, y_{n}\right) \mu_{n}(\mathrm{~d} \omega)\right] \\
& \leq \liminf _{n \rightarrow \infty}\left[\sup _{g \in \mathcal{T}_{p}(\Omega)}\left|\int_{\Omega} g(\omega)\left(-\mu_{n}+\mu_{0}\right)(\mathrm{d} \omega)\right|+\int_{\Omega} m_{i}\left(\omega, y_{n}\right) \mu_{n}(\mathrm{~d} \omega)\right] \\
& \leq \liminf _{n \rightarrow \infty}\left[\zeta_{p}\left(\mu_{n}, \mu\right)+\int_{\Omega} m_{i}\left(\omega, y_{n}\right) \mu_{n}(\mathrm{~d} \omega)\right] \\
& =\liminf _{n \rightarrow \infty} \int_{\Omega} m_{i}\left(\omega, y_{n}\right) \mu_{n}(\mathrm{~d} \omega) \leq 0 .
\end{aligned}
$$

Hence, $y \in H(\mu)$, i.e., $H$ is closed. As $H(\mu)$ is a closed subset of a bounded set $X, H$ is compact-valued, or equivalently, it is upper semicontinuous.

\section{Stability CONDitions}

In this section we investigate the closedness, upper semicontinuity or continuity of solution mappings to stochastic equilibrium problems. From here on, we assume that all assumptions of Theorem 3.10 are satisfied for all $\mu \in M$, and hence by applying Theorem 3.10, the solution sets to such problems are nonempty at the reference points.

We first get the result regarding the closeness of solution set.

Lemma 5.1. Given $\mu \in \mathcal{P}(\Omega)$ and assume that $K$ has closed values. Then, the solution set $S(\mu)$ is a closed set.

Proof. We have

$$
\begin{aligned}
S(\mu) & =\left\{x \in K(\mu): \int_{\Omega} f(\omega, x, y) \mu(\mathrm{d} \omega) \leq 0, \forall y \in K(\mu)\right\} \\
& =K(\mu) \cap\left[\cap_{y \in K(\mu)} \Gamma_{f}(\mu, y)\right] .
\end{aligned}
$$

Combining the closedness of $K(\mu)$ and Lemma 4.1, we conclude that $S(\mu)$ is closed.

Next, we study sufficient conditions for the closedness and continuity properties of the solution mapping $S$ with respect to $(\mathcal{P}(\Omega), \rho)$.

Theorem 5.2. Assume that $K: \mathcal{P}(\Omega) \rightrightarrows X$ has closed values and $f$ is continuous in the second and third arguments for a.e. $\omega$. Then, the solution mapping $S$ is both continuous and closed on $(\mathcal{P}(\Omega), \rho)$. 
Proof. Let $\mu_{0} \in \mathcal{P}(\Omega)$ be arbitrary, using Lemmas 4.2 and 5.1, we conclude that $S$ is closed and lsc at $\mu_{0}$. Next we prove that $S$ is upper semicontinuous at $\mu_{0}$. Suppose, on the contrary, that $S$ is not usc at $\mu_{0}$. Then, there exist an open neighborhood $U$ of $S\left(\mu_{0}\right)$ and a sequence $\left\{\mu_{n}\right\}$ converging to $\mu_{0}$ such that for each $n$, there is $x_{n} \in S\left(\mu_{n}\right) \backslash U$. Since $x_{n} \in S\left(\mu_{n}\right) \subset K\left(\mu_{n}\right)$ for all $n$, we have

$$
d\left(x_{n}, K\left(\mu_{0}\right)\right) \leq \mathcal{H}\left(K\left(\mu_{n}\right), K\left(\mu_{0}\right)\right) \leq \rho\left(\mu_{n}, \mu_{0}\right) .
$$

As $K\left(\mu_{0}\right)$ is closed and $X$ is bounded, for each $n$, there exists $x_{n}^{*} \in K\left(\mu_{0}\right)$ such that

$$
\left\|x_{n}-x_{n}^{*}\right\|=d\left(x_{n}, K\left(\mu_{0}\right)\right) .
$$

Employing the closedness of $K\left(\mu_{0}\right)$ and the boundedness of $X$, we can assume that $\left\{x_{n}^{*}\right\}$ converges to some point $x_{0}$ in $K\left(\mu_{0}\right)$. From (5.1), (5.2) and the fact that $\rho\left(\mu_{n}, \mu_{0}\right) \rightarrow 0$ as $n \rightarrow+\infty$, we have $x_{n} \rightarrow x_{0}$ as $n \rightarrow+\infty$. Since $x_{n} \in S\left(\mu_{n}\right)$,

$$
\int_{\Omega} f\left(\omega, x_{n}, y\right) \mu_{n}(\mathrm{~d} \omega) \leq 0 \forall y \in K\left(\mu_{n}\right) .
$$

Next, we show that $x_{0} \in \Gamma_{f}\left(\mu_{0}, y\right)=\left\{x \in X: \int_{\Omega} f(\omega, x, y) \mu_{0}(\mathrm{~d} \omega) \leq 0\right\}$ for all $y \in K\left(\mu_{0}\right)$. Suppose that there is $y_{0} \in K\left(\mu_{0}\right)$ satisfying $x_{0} \notin \Gamma_{f}\left(\mu_{0}, y_{0}\right)$, i.e., $\int_{\Omega} f\left(\omega, x_{0}, y_{0}\right) \mu_{0}(\mathrm{~d} \omega)>0$. Applying Lemma 4.2, the lower semicontinuity $K$ is obtained, and hence there exists a sequence $\left\{y_{n}\right\}$ such that $y_{n} \in K\left(\mu_{n}\right)$ and $y_{n} \rightarrow y_{0}$. Since $f(\omega, \cdot, \cdot)$ is continuous for a.e. $\omega$ and $\left\{\left(\mu_{n}, x_{n}, y_{n}\right)\right\}$ converges to $\left(\mu_{0}, x_{0}, y_{0}\right)$, we conclude that the corresponding sequence $\left\{\int_{\Omega} f\left(\omega, x_{n}, y_{n}\right) \mu_{n}(\mathrm{~d} \omega)\right\}$ converges to $\int_{\Omega} f\left(\omega, x_{0}, y_{0}\right) \mu_{0}(\mathrm{~d} \omega)>0$. In other words, there is $n_{0} \in \mathbb{N}$ such that for all $n \geq n_{0}, \int_{\Omega} f\left(\omega, x_{n}, y_{n}\right) \mu_{n}(\mathrm{~d} \omega)>0$, which contradicts (5.3). This implies $x_{0} \in \Gamma\left(\mu_{0}, y\right)$ for all $y \in K\left(\mu_{0}\right)$. Hence, $x_{0} \in S\left(\mu_{0}\right) \subset U$, this contradicts the fact that $x_{n} \notin U$ for all $n$, and also $S$ is upper semicontinuous. In summary, $S$ is both continuous and closed on $(\mathcal{P}(\Omega), \rho)$.

Passing to the space $\left(\mathcal{P}_{p}(\Omega), \sigma_{p}\right)$, we also establish a result similar to that of Theorem 5.2.

Theorem 5.3. Assume that the mapping $K: \mathcal{P}_{p}(\Omega) \rightrightarrows X$ has closed values, and for all $\omega, \bar{\omega} \in \Omega$, for all $x, y \in X, f(\omega, \cdot, \cdot)$ is lower semicontinuous for a.e. $\omega$, and

$$
|f(\omega, x, y)-f(\bar{\omega}, x, y)| \leq\|\omega-\bar{\omega}\| \max \left\{1,\|\omega\|^{p-1},\|\bar{\omega}\|^{p-1}\right\} .
$$

Then, the solution mapping $S$ is both continuous and closed on $\left(\mathcal{P}_{p}(\Omega), \sigma_{p}\right)$.

Proof. Let $\mu_{0} \in \mathcal{P}_{p}(\Omega)$, due to Lemmas 4.2 and 5.1, it is necessary to check that $S$ is usc at $\mu_{0}$. If $S$ is not usc at $\mu_{0}$, then there are an open neighborhood $U$ of $S\left(\mu_{0}\right)$ in $X$ and a sequence $\left\{\mu_{n}\right\}$ converging to $\mu_{0}$ such that for each $n$, there exists $x_{n} \in S\left(\mu_{n}\right)$ but $x_{n} \notin U$. By the proof of Theorem 5.2, $\left\{x_{n}\right\}$ converges to $x_{0} \in K\left(\mu_{0}\right)$, we prove that

$$
\int_{\Omega} f\left(\omega, x_{0}, y\right) \mu_{0}(\mathrm{~d} \omega) \leq 0, \forall y \in K\left(\mu_{0}\right) .
$$

By Lemma 4.2, for each $y \in K\left(\mu_{0}\right)$, there exists $y_{n} \in K\left(\mu_{n}\right)$ such that $y_{n} \rightarrow y$. Combining (5.4) with the lower semicontinuity of $f(\omega, \cdot, \cdot)$ for a.e. $\omega$ and Fatou's lemma, we imply that

$$
\begin{aligned}
\int_{\Omega} f\left(\omega, x_{0}, y\right) \mu_{0}(\mathrm{~d} \omega) & \leq \liminf _{n \rightarrow \infty} \int_{\Omega} f\left(\omega, x_{n}, y_{n}\right) \mu_{0}(\mathrm{~d} \omega) \\
& \leq \liminf _{n \rightarrow \infty}\left[\left|\int_{\Omega} f\left(\omega, x_{n}, y_{n}\right)\left(-\mu_{n}+\mu_{0}\right)(\mathrm{d} \omega)\right|+\int_{\Omega} f\left(\omega, x_{n}, y_{n}\right) \mu_{n}(\mathrm{~d} \omega)\right] \\
& \leq \liminf _{n \rightarrow \infty}\left[\sup _{g \in \mathcal{T}_{p}(\Omega)}\left|\int_{\Omega} g(\omega)\left(-\mu_{n}+\mu_{0}\right)(\mathrm{d} \omega)\right|+\int_{\Omega} f\left(\omega, x_{n}, y_{n}\right) \mu_{n}(\mathrm{~d} \omega)\right]
\end{aligned}
$$




$$
\begin{aligned}
& \leq \liminf _{n \rightarrow \infty}\left[\sigma_{p}\left(\mu_{n}, \mu_{0}\right)+\int_{\Omega} f\left(\omega, x_{n}, y_{n}\right) \mu_{n}(\mathrm{~d} \omega)\right] \\
& =\liminf _{n \rightarrow \infty} \int_{\Omega} f\left(\omega, x_{n}, y_{n}\right) \mu_{n}(\mathrm{~d} \omega) \leq 0 .
\end{aligned}
$$

So, $x_{0} \in S\left(\mu_{0}\right) \subset U$ that is incorrect as $x_{n} \notin U$ for all $n$. The proof follows.

In the special case, $K: \mathcal{P}_{p}(\Omega) \rightrightarrows X$ is identified by (4.1), we obtain sufficient conditions for the solution set to (SEP) to be closed and upper semicontinuous with respect to the Fortet-Mourier metric as follows.

Theorem 5.4. Assume that the mapping $K$ is identified by (4.1), and assume further that $f(\omega, \cdot, y)$ is lower semicontinuous for any $(\omega, y) \in \Omega \times X$ and the multivalued mapping $T:\left(\mathcal{P}_{p}(\Omega), \zeta_{p}\right) \rightrightarrows X$ is given by

$$
T(\mu)=\left\{x \in X: \int_{\Omega} f(\omega, x, y) \mu(\mathrm{d} \omega) \leq 0, \forall y \in K(\mu)\right\}
$$

is closed. Then, the solution mapping $S$ is both closed and upper semicontinuous on $\left(\mathcal{P}_{p}(\Omega), \zeta_{p}\right)$.

Proof. Applying Lemma 4.4, $K$ is closed. It is clear that

$$
S(\mu)=K(\mu) \cap T(\mu) .
$$

Since $f(\omega, \cdot, y)$ is lower semicontinuous, $T(\cdot)$ is closed, and hence $S(\cdot)$ is closed. By the boundeness of $X$, we imply that $S$ is closed with compact values, and hence it is upper semicontinuous.

Remark 5.5. Applying Fatou's lemma, one can prove that the mapping $T$ defined in Theorem 5.4 is closed if $K$ is continuous, and $f(\omega, \cdot, \cdot)$ is lower semicontinuous for a.e. $\omega \in \Omega$.

\section{Applications}

In this section, as examples, we apply the main results to two special cases of the stochastic equilibrium problem depending on parameters of a probability measure. Based on a modified version of the KKM-Fan theorem and new metrics proposed in Section 4, we study qualitative properties of solutions to such special cases, and hence our approaches and results are different from the existing ones in the literature. Let $X, \Omega$, $\mathcal{P}(\Omega), \mathcal{P}_{p}(\Omega)$ be defined as the previous sections.

\subsection{Stochastic optimization problems}

Let $F, m_{i}: \Omega \times X \rightarrow \overline{\mathbb{R}}$ be mappings such that $F(\cdot, x)$ and $m_{i}(\cdot, x)$ are measurable for all $x \in X$ and $i=1,2, \ldots, n$. Let $K: M \rightrightarrows X$ be given by

$$
K(\mu)=\left\{x \in X: \int_{\Omega} m_{i}(\omega, x) \mu(\mathrm{d} \omega) \leq 0, \quad i=1,2, \ldots, n\right\} .
$$

We consider the parametric stochastic optimization problem studied in [24]:

$$
\left(\min _{x \in K(\mu)} \mathbb{E}_{\mu}[F(\omega, x)]=\min _{x \in K(\mu)} \int_{\Omega} F(\omega, x) \mu(\mathrm{d} \omega) .\right.
$$

Setting $f(\omega, x, y)=F(\omega, x)-F(\omega, y)$, then the problem $\left(\mathrm{SOP}_{\mu}\right)$ becomes a special case of the problem $\left(\mathrm{SEP}_{\mu}\right)$.

For $\mu \in M$, the optimal value of $\left(\mathrm{SOP}_{\mu}\right)$ at parameter $\mu$ is

$$
v(\mu)=\min _{x \in K(\mu)} \int_{\Omega} F(\omega, x) \mu(\mathrm{d} \omega),
$$


and the solution mapping $S_{1}$ to $\left(\mathrm{SOP}_{\mu}\right)$ is identified by

$$
S_{1}(\mu)=\left\{x \in K(\mu): \int_{\Omega} F(\omega, x) \mu(\mathrm{d} \omega)=v(\mu)\right\} .
$$

Now we equip $\mathcal{P}_{p}(\Omega)$ with a metric $\sigma_{p}^{\prime}$ as follows.

For $\mu, \nu \in \mathcal{P}_{p}(\Omega)$,

$$
\sigma_{p}^{\prime}(\mu, \nu)=\max \left\{\sup _{K^{\prime} \in \mathcal{K}} h\left(K^{\prime}(\mu), K^{\prime}(\nu)\right), \sup _{g \in \mathcal{T}_{p}(\Omega)}\left|\int_{\Omega} g(\omega)(\mu-\nu)(\mathrm{d} \omega)\right|\right\} .
$$

Obviously, for all $\mu, \nu \in \mathcal{P}_{p}(\Omega)$, we have

$$
\sigma_{p}^{\prime}(\mu, \nu) \leq \sigma_{p}(\mu, \nu) \leq 2 \sigma_{p}^{\prime}(\mu, \nu) .
$$

Employing the results of Section 5, we introduce sufficient conditions of the existence and stability for the problem $\left(\mathrm{SOP}_{\mu}\right)$.

Corollary 6.1. For $\left(\mathrm{SOP}_{\mu}\right)$, assume that

(i) $K: \mathcal{P}_{p}(\Omega) \rightrightarrows X K$ has nonempty, convex and compact values;

(ii) $F$ is continuous and quasiconvex in the second argument for a.e. $\omega \in \Omega$;

(iii) $F$ is measurable in the first argument, and for all $\omega, \bar{\omega} \in \Omega, x \in X$,

$$
|F(\omega, x)-F(\bar{\omega}, x)| \leq \frac{1}{2}\|\omega-\bar{\omega}\| \max \left\{1,\|\omega\|^{p-1},\|\bar{\omega}\|^{p-1}\right\} .
$$

Then, the solution mapping $S_{1}$ identified by $(6.1)$ is closed, continuous and nonempty-valued on $\left(\mathcal{P}_{p}(\Omega), \sigma_{p}\right)$ and $\left(\mathcal{P}_{p}(\Omega), \sigma_{p}^{\prime}\right)$, and further, for all $\mu, \nu \in \mathcal{P}_{p}(\Omega)$,

$$
|v(\mu)-v(\nu)| \leq \sigma_{p}^{\prime}(\mu, \nu)
$$

Proof. Setting $f(\omega, x, y)=F(\omega, x)-F(\omega, y)$ for all $\omega \in \Omega$, and $x, y \in X$, then $\left(\mathrm{SOP}_{\mu}\right)$ becomes a special of the problem $\left(\mathrm{SEP}_{\mu}\right)$. Now we check that all assumptions of Theorem 3.7 hold true. Obviously, the assumption (ii) of this theorem is satisfied as $F$ is quasiconvex in the second argument for a.e. $\omega \in \Omega$.

Because $\Omega$ is a Borel set and $F$ is lower semicontinuous, Theorem 14.31 of [28] implies that $f(\cdot, \cdot, y)$ is random lower semicontinuous for each $y \in K(\mu)$. Due to Theorem 3.10, the first assumption is also fulfilled. Applying Theorem 3.7, we conclude that the problem $\left(\mathrm{SOP}_{\mu}\right)$ has solutions for all $\mu$.

Applying Theorem 5.3 for $f(\omega, x, y)=F(\omega, x)-F(\omega, y)$, the conclusion (a) is obtained.

For the conclusion (b), employing (6.2), the closedness and continuity of $S_{1}$ on $\left(\mathcal{P}_{p}(\Omega), \sigma_{p}^{\prime}\right)$ are derived by that ones of $S_{1}$ on $\left(\mathcal{P}_{p}(\Omega), \sigma_{p}\right)$. Now, for all $\mu, \nu \in \mathcal{P}_{p}(\Omega)$, let $x^{\prime} \in S_{1}(\mu)$ and $x^{\prime \prime} \in S_{1}(\nu)$, we have

$$
\begin{aligned}
|v(\mu)-v(\nu)| & \leq \max \left\{\int_{\Omega} F\left(\omega, x^{\prime}\right)(\mu-\nu)(\mathrm{d} \omega), \int_{\Omega} F\left(\omega, x^{\prime}\right)(\nu-\mu)(\mathrm{d} \omega)\right\} \\
& \leq \sup _{g \in \mathcal{T}_{p}(\Omega)}\left|\int_{\Omega} g(\omega)(\mu-\nu)(\mathrm{d} \omega)\right| \\
& \leq \max \left\{\sup _{K^{\prime} \in \mathcal{K}} h\left(K^{\prime}(\mu), K^{\prime}(\nu)\right), \sup _{g \in \mathcal{T}_{p}(\Omega)}\left|\int_{\Omega} g(\omega)(\mu-\nu)(\mathrm{d} \omega)\right|\right\} \\
& =\sigma_{p}^{\prime}(\mu, \nu) .
\end{aligned}
$$

Hence, the proof is complete. 


\subsection{Optimization problem with probabilistic constraint}

Let $X$ be a convex, closed and bounded subset of $\mathbb{R}^{n}, g: X \rightarrow \mathbb{R}^{k}$ be a mapping, and let $\kappa: X \rightarrow \mathbb{R}^{m}$ be a continuous mapping and $\xi: \Omega \rightarrow \mathbb{R}^{m}$ be a mapping with $m$-dimensional stochastic vector values. Let $r$ be fixed, we consider the following parametric optimization problem with stochastic constraint.

$\left(\mathrm{OP}_{\mu}\right) \quad \min \{g(x): x \in X, \mu(\{\omega \in \Omega: \kappa(x) \geq \xi(\omega)\}) \geq r\}$.

Setting

$$
\begin{aligned}
f(\omega, x, y) & =g(x)-g(y), \\
K(\mu) & =\{x \in X: \mu(\{\omega \in \Omega: \kappa(x) \geq \xi(\omega)\}) \geq r\},
\end{aligned}
$$

then $\left(\mathrm{OP}_{\mu}\right)$ becomes a special case of $\left(\mathrm{SEP}_{\mu}\right)$. Assigning

$$
\Delta_{\mu}(\kappa(x))=\mu(\omega \in \Omega: \kappa(x) \geq \xi(\omega)) .
$$

As $\kappa$ is continuous, $K(\mu)=\left\{x \in X: \Delta_{\mu}(\kappa(x)) \geq r\right\}$ is closed. Therefore, the distance $\rho^{\prime \prime}$ on $\mathcal{P}(\Omega)$ defined as

$$
\rho^{\prime \prime}(\mu, \nu)=\sup _{K^{\prime} \in \mathcal{K}} \mathcal{H}\left(K^{\prime}(\mu), K^{\prime}(\nu)\right) \forall \mu, \nu \in \mathcal{P}(\Omega)
$$

is a metric on $\mathcal{P}(\Omega)$.

Similar to the first one, now we discuss existence and stability conditions of the solutions to the problem $\left(\mathrm{OP}_{\mu}\right)$.

Corollary 6.2. For $\left(\mathrm{OP}_{\mu}\right)$, assume that

(i) the set $\mu(\{\omega \in \Omega: \kappa(x) \geq \xi(\omega)\}) \geq r\}$ is nonempty, convex and compact;

(ii) $g$ is continuous and quasiconvex.

Then, the problem $\left(\mathrm{OP}_{\mu}\right)$ is feasible for each $\mu$ and its solution mapping defined by

$$
S_{2}(\mu)=\operatorname{argmin}\left\{g(x): x \in X, \Delta_{\mu}(\kappa(x)) \geq r\right\}
$$

is both closed and continuous on $\left(\mathcal{P}(\Omega), \rho^{\prime \prime}\right)$.

Proof. Setting

$$
\begin{aligned}
K(\mu) & =\{x \in X: \mu(\{\omega \in \Omega: \kappa(x) \geq \xi(\omega)\}) \geq r\}\}, \\
f(\omega, x, y) & =g(x)-g(y), \forall \omega \in \Omega, \forall x, y \in K(\mu),
\end{aligned}
$$

then $\left(\mathrm{OP}_{\mu}\right)$ is also a special case of $\left(\mathrm{SEP}_{\mu}\right)$. Using the similar arguments as that of the proof for Corollary 6.1, we conclude that the solution set of $\left(\mathrm{OP}_{\mu}\right)$ is nonempty for each $\mu$. Combining Theorem 5.2 and the fact that $\rho^{\prime \prime}(\mu, \nu)=\rho(\mu, \nu)$ for all $\mu, \nu \in \mathcal{P}(\Omega)$, we obtain the last conclusion of Corollary 6.2.

Acknowledgements. The authors would like to thank the two referees for their valuable remarks and suggestions that helped us significantly improve the paper. This research is funded by Vietnam National Foundation for Science and Technology Development (NAFOSTED) under grant number 101.01-2020.11.

\section{REFERENCES}

[1] S.Z. Alparslan Gök, Some results on cooperative interval games. Optimization 63 (2014) 7-13.

[2] L.Q. Anh and T.Q. Duy, Tykhonov well-posedness for lexicographic equilibrium problems. Optimization 65 (2016) $1929-1948$.

[3] B. Ankenman, B.L. Nelson and J. Staum, Stochastic Kriging for simulation metamodeling. Oper. Res. 58 (2010) $371-382$.

[4] A. Barbagallo and G. Scilla, Stochastic weighted variational inequalities in non-pivot Hilbert spaces with applications to a transportation model. In: New trends in optimization and variational analysis and applications; an international conference in honor of Prof. Michel Théra for his 70th birthday, Quy Nhon, Viet Nam, 2016 December 7-10 (2016). 
[5] E. Blum and W. Oettli, From optimization and variational inequalities to equilibrium problems. Math. Student. 63 (1994) $123-145$.

[6] G.M. Cho, Stability of the multiple objective linear stochastic programming problems. Bull. Korean Math. Soc. 32 (1995) $287-296$.

[7] X. Chen, R.J.-B. Wets and Y. Zhang, Stochastic variational inequalities: residual minimization smoothing sample average approximations. SIAM J. Optim. 22 (2012) 649-673.

[8] A. Evgrafov and M. Patriksson, On the existence of solutions to stochastic mathematical programs with equilibrium constraints. J. Optim. Theory Appl. 121 (2004) 65-76.

[9] K. Fan, A minimax inequality and applications, In: Inequality III, edited by O. Shisha. Academic Press, New York, NY (1972) 103-113.

[10] K. Fan, Some properties of convex sets related to fixed point theorems. Math. Annalen. 266 (1984) 519-537.

[11] A.P. Farajzadeh, On the symmetric vector quasiequilibrium problems. J. Math. Anal. Appl. 322 (2006) $1099-1110$.

[12] R. Henrion and W. Römisch, Metric regular and quantitative stability in stochastic programs with probabilistic constraints. Math. Program. 84 (1999) 55-88.

[13] R. Henrion and W. Römisch, Problem-based optimal scenario generation and reduction in stochastic programming. To appear in: Math. Program. (2018) DOI: 10.1007/s10107-018-1337-6.

[14] D.R. Jones, M. Schonlau and W.J. Welch, Efficient global optimization of expensive black-box functions. J. Global Optim. 13 (1998) 455-492.

[15] V. Kaňková, Stability in the stochastic programming. Kybernetika 14 (1978) 339-349.

[16] V. Kaňková, On stability in two-stage stochastic nonlinear programming, edited by P. Mandl and M. Hušková. In: Vol. 5 of Asymptotic Statistics. Springer, Berlin (1994) 329-340.

[17] V. Kaňková, On the stability in stochastic programming-generalized simple recourse problems. Informatica 5 (1994) 55-78.

[18] B. Knaster, C. Kuratowski and S. Mazurkiewicz, Ein Beweis des Fixpunktsatzes für n-dimensionale Simplexe. Fundam. Math. 14 (1929) 132-137.

[19] L.D. Muu, Stability property of a class of variational inequalities. Math Operationsforsch Statist. Ser. Optim. 15 (1984) 347-351.

[20] L.D. Muu and W. Oettli, Convergence of an adaptive penalty scheme for finding constrained equilibria. Nonlinear Anal. 18 (1992) 1159-1166.

[21] A. Nemirovski, A. Juditsky, G. Lan and A. Shapiro, Robust stochastic approximation approach to stochastic programming. SIAM J. Optim. 19 (2009) 1574-1609.

[22] M. Patriksson and L. Wynter, Stochastic mathematical programs with equilibrium constraints. Oper. Res. Lett. 25 (1999) 159-167.

[23] S.T. Rachev, Probability Metrics and the Stability of Stochastic Models. Wiley, Chichester, UK (1991).

[24] S.T. Rachev and W. Römisch, Quatitative stability in stochastic programming: the method of probability metrics. Math. Oper. Res. 27 (2002) 792-818.

[25] U. Ravat and U.V. Shanbhag, On the characterization of solution sets of smooth and nonsmooth convex stochastic nash games. SIAM J. Optim. 21 (2011) 1168-1199.

[26] U. Ravat and U.V. Shanbhag, On the existence of solutions to stochastic quasi-variational inequality and complementarity problems. Math. Program. 165 (2017) 291-330.

[27] R.T. Rockafellar and R.J.-B. Wets, Measures as Lagrange multipliers in multistage stochastic programming. J. Math. Anal. Appl. 60 (1977) 301-313.

[28] R.T. Rockafellar and R.J.-B. Wets, Variational Analysis. Springer, Berlin (1998).

[29] W. Römisch, Stability of stochastic programming problems, In: Stochastic Programming, edited by A. Rusczyński and A. Shapiro. Vol. 10 of Handbooks in Operations Research and Management Science (2003) 483-554.

[30] W. Römisch and R.J.-B. Wets, Stability of $\epsilon$-approximate solutions to convex stochastic programs. SIAM J. Optim. 18 (2007) 961-979.

[31] A. Rusczyński and A. Shapiro, Stochastic programming models, Stochastic Programming, edited by A. Rusczyński and A. Shapiro. In Vol. 10 of Handbooks in Operations Research and Management Science (2003) 1-64.

[32] A. Rusczyński and A. Shapiro, Optimality and duality in stochastic programming, Stochastic Programming. edited by A. Rusczyński and A. Shapiro. In Vol. 10 of Handbooks in Operations Research and Management Science (2003) 65-139.

[33] U.V. Shanbhag, Stochastic variational inequality problems: applications, analysis, and algorithms. TUTORIALS Oper. Res. 2013 (2013) 71-107.

[34] A. Shapiro, Stochastic programming approach to optimization under uncertainty. Math. Program Ser. B. 112 (2008) 183-220.

[35] A. Shapiro and H. Xu, Stochastic mathematical programs with equilibrium constraints, modelling and sample average approximation. Optimization 57 (2008) 395-418.

[36] W. Takahashi, Nonlinear variational inequalities and fixed point theorems. J. Math. Soc. Jpn. 28 (1976) $168-181$.

[37] P. Terán, On consistency of stationary points of stochastic optimization problems in a Banach space. J. Math. Anal. Appl. 363 (2010) 569-578.

[38] R. Wangkeeree and U. Kamraksa, Existence theorems and iterative approximation methods for generalized mixed equilibrium problems for a countable family of nonexpansive mappings. J. Global Optim. 54 (2012) 27-46. 
[39] G.-W. Weber, A. Kruger, J.E. Martínez-Legaz, B. Mordukhovich and L. Sakalauskas, Special Issue on recent advances in continuous optimization on the occasion of the 25th European conference on Operational Research (EURO XXV 2012). Optimization 63 (2014) 1-166.

[40] R.J.-B. Wets, Stochastic programming, edited by G.L. Nemhauser. In Vol. 1 of Handbooks in Operations Research and Management Science (1989) 573-629.

[41] $\mathrm{H}$. Xu, Uniform exponential convergence of sample average random functions under general sampling with applications in stochastic programming. J. Math. Anal. Appl. 368 (2010) 692-710.

[42] H. Xu, Sample average approximation methods for a class of stochastic variational inequality problems. Asia Pac. J. Oper. Res. 27 (2010) 103-119.

[43] H. Xu and J.J. Ye, Necessary optimality conditions for two-stage stochastic programs with equilibrium constraints. SIAM J. Optim. 20 (2010) 1685-1715.

[44] H. Xu and D. Zhang, Stochastic Nash equilibrium problems: sample average approximation and applications. Comput. Optim. Appl. 55 (2013) 597-645.

[45] J. Zhang, Y. Ma, T. Yang and L. Liu, Estimation of the Pareto front in stochastic simulation through stochastic Kriging. Simul. Model Pract. Theory. 79 (2017) 69-86. 